\title{
Delayed-type retrobulbar hematoma caused by low temperature after reconstruction of inferior blow- out fracture
}

Da Woon Lee ${ }^{1}$, Tae Hyung Kim², Hwan Jun Choi ${ }^{1}$, Syeo Young Wee ${ }^{2}$

${ }^{1}$ Department of Plastic and Reconstructive Surgery, Soonchunhyang University Cheonan Hospital, Cheonan; ${ }^{2}$ Department of Plastic and Reconstructive Surgery, Soonchunhyang University Gumi Hospital, Gumi, Korea
Retrobulbar hemorrhage is a disastrous condition that can lead to permanent blindness. As such, rapid diagnosis and treatment are critical. Here, we report a patient who presented with retrobulbar hemorrhage following an orbital floor fracture. Restoration of inferior orbital wall with porous polyethylene implant was underwent. Four days after the orbital floor reconstruction, the patient smoked a cigarette outdoors in $-3^{\circ} \mathrm{C}$ weather. Cold temperature and smoking caused an increase in his systemic blood pressure. The elevated blood pressure increased intraorbital pressure to the extent of causing central retinal artery occlusion and exacerbated oozing. During exploratory surgery, $3 \mathrm{~mL}$ of hematoma and diffuse oozing without arterial bleeding were observed. Prompt diagnosis and treatment prevented vision impairment. Few studies have reported on the risk factors for retrobulbar hemorrhage. This case showed that daily activities, such as exposure to cold weather or tobacco smoking, could be risk factors for retrobulbar hemorrhage.

Keywords: Blindness / Orbital fracture / Postoperative complication / Retrobulbar hemorrhage

\section{INTRODUCTION}

Retrobulbar hemorrhage is a rare, but potentially disastrous condition because it can cause permanent visual impairment and blindness. It is known to occur mainly after a periorbital trauma or orbital fracture reconstruction, but it can also be caused by periorbital surgery, such as blepharoplasty, or even circumbulbar anesthesia [1].

The incidence of retrobulbar hemorrhage after orbital fracture reconstruction is $0.45 \%$ to $0.6 \%[2,3]$. Facial fracture associatedblindness is the most common cause of traumatic visual loss

Correspondence: Hwan Jun Choi

Department of Plastic and Reconstructive Surgery, Soonchunhyang University Cheonan Hospital, 31 Suncheonhyang 6-gil, Dongnam-gu, Cheonan 31151, Korea E-mail: iprskorea@gmail.com

This work was supported by the National Research Foundation of Korea (NRF) grant funded by the Korea government (MSIT) (2020R1A2C1100891), and was supported by Soonchunhyang University Research Fund.

Received February 17, 2021 / Revised April 14, 2021 / Accepted April 20, 2021 following traumatic optic neuropathy and direct eyeball injury $[3,4]$.

Retrobulbar hemorrhage after orbital fracture reconstruction is known to occur within 24 hours of surgery [5]. The optic nerve and retina, which play important roles in visual acuity, can only withstand 60-90 minutes of elevated intraocular pressure due to retrobulbar hemorrhage $[1,3,6]$. After that, permanent damage of the optic nerve and retina leading to permanent vision loss is likely. Therefore, rapid diagnosis and treatment are critical.

The mechanism of retrobulbar hemorrhage is related to bleeding caused by damage to the infraorbital or the anterior and posterior ethmoidal arteries during an operation that subsequently causes persistent bleeding after wound closure [1-3]. The treatment of retrobulbar hemorrhage is surgical decompression within 24 hours after the diagnosis [1-3]. Although the mechanism and treatment of retrobulbar hemorrhage are known, studies that reported the risk factors for retrobulbar he- 
matomas are few. Here, we present a case of retrobulbar hemorrhage after orbital wall fracture reconstruction and discuss the risk factors associated with retrobulbar hemorrhage.

\section{CASE REPORT}

A 34-year-old man without any underlying disease was transferred to our hospital for swelling of the left periorbital area after being hit by a baseball. An orbital computed tomography (CT) scan showed an orbital floor fracture (Fig. 1A). Reconstruction of the depressed orbital floor with porous polyethylene implant (Medpor; Porex Corp., College Park, GA, USA) and insertion of a negative pressure drainage tube were performed. Before surgery, ophthalmologists confirmed the absence of abnormalities on the left eye. No complications were observed until the third postoperative day. On the 4th postoperative day, the patient went outside and smoked a cigarette. It was winter at the time, and the temperature was $-3^{\circ} \mathrm{C}$. A few hours later, the patient suddenly presented with severe eye pain, edema, and chemosis. Relevant medical information including vital signs and smoking history are presented in Table 1. The patient's blood pressure and pulse rate were slightly increased after the onset of these symptoms. Unilateral proptosis and a dilated pupil were observed and the patient's visual acuity on his left eye decreased from 20/20 initially to hand motion. A diagnostic enhanced facial CT scan revealed a $22 \times 16 \times 12 \mathrm{~mm}$ retrobulbar hematoma and $25 \mathrm{~mm}$ of exophthalmos (Fig. 1B-D). Since the signs of retrobulbar hematoma were unequivocal, a decompression proce- dure was immediately performed without measuring the intraocular pressure. Bedside decompression via suture removal was performed immediately. Medical treatment including intravenous steroids and 20\% mannitol were administered simultaneously. Approximately 2 hours after the onset of initial symptoms, emergency exploration under general anesthesia was conducted (Fig. 2A). Intraoperatively, $3 \mathrm{~mL}$ of hematoma as a form of fluid collection was evacuated from the inferior portion of the orbital cavity near the previously inserted Medpor. The quantity of hematoma was also measured as $3.025 \mathrm{~mL}$ using the picture archiving and communication system software, Dejaview (Dongeun IT, Bucheon, Korea). Active arterial bleeding was not seen, but diffuse oozing was observed. Meticulous he-

Table 1. Patient and medical information before and after retrobulbar hemorrhage

\begin{tabular}{lrrrc}
\hline Medical information & POD 1 & POD 2 & POD 3 & $\begin{array}{c}\text { Right after the } \\
\text { symptom begun }\end{array}$ \\
\hline Vital sign ${ }^{\text {a) }}$ & 110 & 110 & 110 & 130 \\
SBP $(\mathrm{mmHg})$ & 60 & 70 & 70 & $80-90$ \\
DBP $(\mathrm{mmHg})$ & 68 & 64 & 68 & $64-78$ \\
$\quad$ Pulse rate (beats/min) & 36.3 & 36.5 & 36.5 & 36.1 \\
$\quad$ Temperature $\left({ }^{\circ} \mathrm{C}\right)$ & 2 & 2 & 1 & - \\
$\begin{array}{l}\text { Negative suction drainage } \\
\text { amount (mL) }\end{array}$ & & & & \\
Smoking history & & Current smoker ${ }^{\mathrm{b})}$ & \\
\hline
\end{tabular}

POD, postoperative day; SBP, systolic blood pressure; DBP, diastolic blood pressure.

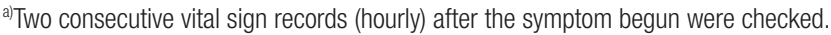
It was all the records before the emergency operation was performed; ${ }^{\text {b) }} 0.5$ pack day $\times 10$ years $=5$ pack-year.
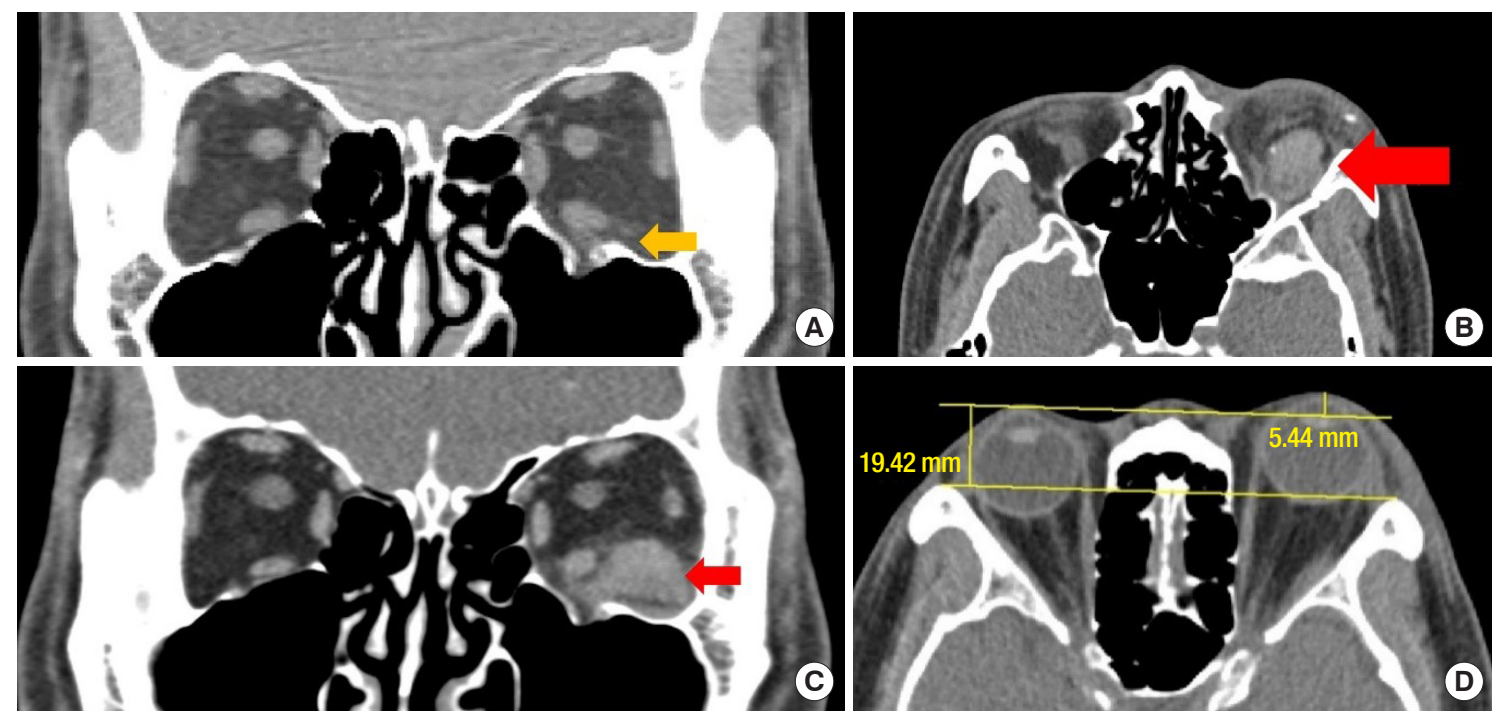

Fig. 1. Computed tomography scan of the patient. (A) Initial computed tomography scan showing coronal view of the orbital floor fracture. Infraorbital wall fracture was noted (yellow arrow). (B, C) Emergency enhanced facial computed tomography scan showing a $22 \times 16 \times 12$ mm retrobulbar hematoma (red arrow) near the Medpor. High attenuation at posterior aspect of lesion was noted, indicating postoperative hematoma. (D) Exophthalmos of $25 \mathrm{~mm}$ on the left eye due to a retrobulbar hematoma. 

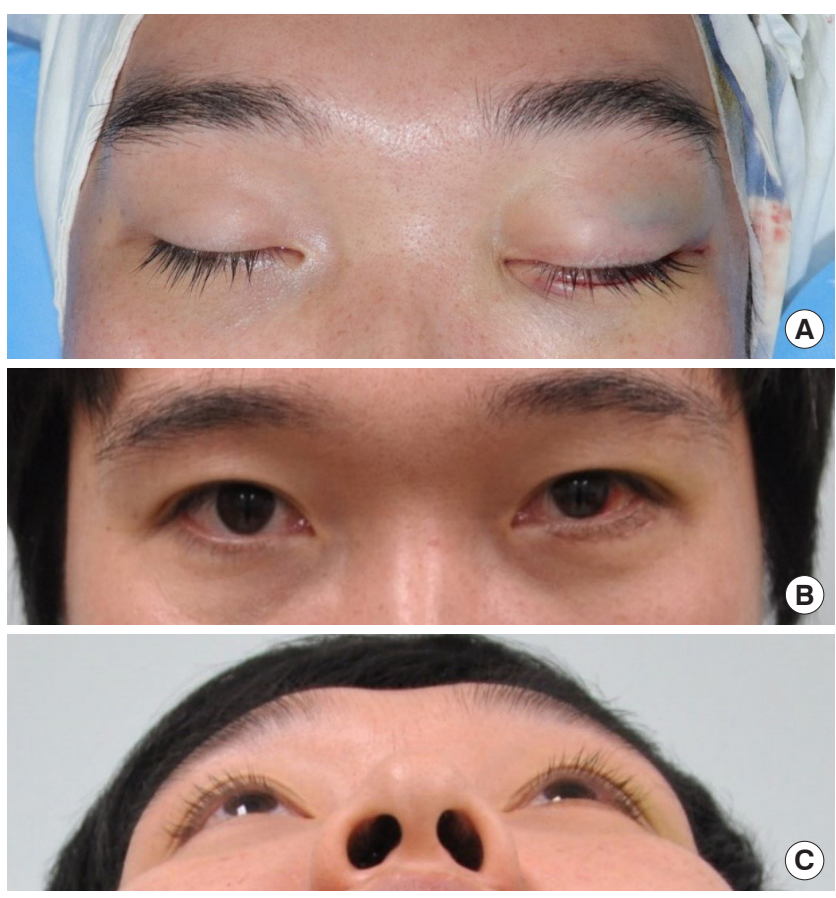

Fig. 2. Photographic findings of the patient. (A) The patient presented with proptosis and periorbital swelling after tobacco smoking in cold weather. Bedside suture removal was already done. (B) No complications, such as decreased visual acuity, diplopia, or exophthalmos on the postoperative follow-up at 4 weeks. (C) Worm's eye view at 4 th postoperative week.

mostasis was performed after removal of the Medpor. A negative pressure drainage tube was inserted, and the wound was closed. No abnormal findings were observed during the postoperative ophthalmic examination. Subsequently, visual acuity on his left eye was restored to $20 / 20$. On the 4 th postoperative day, the drainage tube was removed. On the seventh day, the patient was discharged. No complications were observed in subsequent follow-up examinations at 1,2, and 4 weeks, and protrusion of the left eye also resolved (Fig. 2B and C).

\section{DISCUSSION}

The clinical features, diagnosis, and management of the retrobulbar hemorrhage are well described in the literature. Patients present with severe periorbital pain, proptosis, mydriasis, tense hardening of the eyeball, decreased visual acuity, and extraocular muscle movement limitations [1,3]. Diagnosis is confirmed by CT scan. However, if retrobulbar hemorrhage is suspected, a CT scan for diagnostic purposes should not delay treatment initiation [3]. There are few reports of recovery following vision loss due to retrobulbar hemorrhage from orbital fracture repair [4]. To minimize vision loss, it is necessary to diagnose and treat quickly.
Table 2. A summary of patients with blow-out fractures during 2010 to 2019

\begin{tabular}{lc}
\hline Variable & Value \\
\hline Total number & 371 \\
Average age (range, yr) & $35(1-75)$ \\
Retrobulbar hemorrhage & 1 \\
Minor complications & 31 \\
Winter season & b) \\
Smoking & 104 \\
Smoking + winter season & 238 \\
\hline
\end{tabular}

${ }^{a}$ Minor complications including subconjunctival hemorrhage, swelling, itching sensation, and such; ${ }^{b}$ Months (January, February, and December) including days lower than $0^{\circ} \mathrm{C}$ are classified into winter season.

Four days after the orbital floor fracture reconstruction, the patient smoked a cigarette in cold weather and suddenly developed symptoms suggestive of retrobulbar hemorrhage. As retrobulbar hemorrhage was suspected, we immediately started bedside decompression via suture removal and medications. Simultaneously, the diagnosis was confirmed by an enhanced orbital CT scan. Decompression was performed through surgical exploration within 2 hours of symptoms onset, and vision loss was avoided.

Park et al. [6] reported two cases of retrobulbar hemorrhage in a span of 6 years. They described that a negative pressure drainage system is effective in retrobulbar hemorrhage prevention [6], which we followed. In our institution, negative pressure drainage systems were used in 371 patients between 2010 to 2019. Of these patients, this is the first case of retrobulbar hemorrhage (Table 2). The drainage cannula was probably not functioning properly in this case. Therefore, close observation, including visual examination, is essential after using negative pressure drainage systems during periorbital surgery. Under the normal protocol, drain function is checked every 8 hours by our nurses to ensure that the drain is working properly. Closer observations may be needed for the patients with risk factors such as those suggested in this report. Several studies on delayed retrobulbar hemorrhage have suggested that the accumulation of blood could be one of the reasons for delayed complications $[1,7,8]$. The implant placed in the orbital area may have also impeded fluid drainage. In this case, the malfunction of the drainage system was thought to be the reason. The possibility of drain malfunction alone causing retrobulbar hemorrhage is unlikely unless any other aggravating risk factors such as cold weather is present [7]. Moreover, the medical records showed that the drain system was functioning properly (Table 1).

Retrobulbar hemorrhage after orbital fracture repair is mainly caused by iatrogenic vessel injury [3]. Excessive intraoperative manipulation of the orbital tissues causes bleeding of the zygo- 
maticotemporal vessels, anterior and posterior ethmoidal arteries, or infraorbital arteries [1]. If hemostasis is not adequately achieved, a hematoma develops postoperatively. Persistent hemorrhage and hematoma formation lead to an increase in orbital cavity volume. However, because the orbital cavity is enclosed by rigid bony structures and a fascial envelope, even a small increase in volume causes an increase in intraorbital pressure [1-3]. This results in venous compression and interferes with the intraorbital blood flow, creating a vicious cycle that further increases intraorbital pressure. This is referred to as orbital compartment syndrome $[1,2,6]$. Due to sustained increased intraorbital pressure, central retinal artery occlusion develops, resulting in ischemic injury of the optic nerve or the retina, and ultimately, vision loss $[1,2,6]$.

In this case, considering that diffuse oozing without arterial bleeding was found intraoperatively, the hematoma due to postoperative oozing did not increase intraorbital pressure to the extent of causing central retinal arterial occlusion. In addition, no other factors, except smoking and cold weather, could have affected the situation, since the patient was young and had no medical history relating to ophthalmology and other body systems. Cold temperature and tobacco smoking, as in our case, cause vasoconstriction, which results in increased systemic blood pressure $[9,10]$. An increase in temperature from $10^{\circ} \mathrm{C}$ to $25^{\circ} \mathrm{C}$ causes blood pressure to change sharply, but blood pressure tends to remain relatively steady below $10^{\circ} \mathrm{C}$ [10]. In our case, the cold environment and large temperature difference between the ward and outdoor could possibly have increased the blood pressure. The increased blood pressure in turn increased the intraorbital pressure, to an extent that resulted in occlusion of the central retinal artery. Additionally, the elevated blood pressure may have exacerbated the diffuse oozing, thereby preventing the elevated intraorbital pressure from exiting the vicious cycle.

There are few studies regarding risk factors or prevention of retrobulbar hemorrhage. Son et al. reported that anticoagulants, though not acetylsalicylic acid, significantly increase the risk of retrobulbar hemorrhage [11,12]. Contrarily, Matharu et al. [13] reported a case of retrobulbar hemorrhage in a patient on dualantiplatelet therapy (acetylsalicylic acid and prasugrel). The authors emphasized the importance of investigating the medical history for antithrombotic agent usage in patients with orbital fractures or retrobulbar injections $[11,13]$. However, our patient did not have such a medical history. A previous report stressed that actions causing intraorbital pressure elevation, such as Valsalva manipulation, coughing and vomiting, should be avoided $[1,6]$. Our case demonstrated that not only these actions, but daily activities such as exposure to cold weather and tobacco smoking, may be risk factors for retrobulbar hemorrhage. Our retrospective chart reviews showed that only one retrobulbar hematoma case was reported over 10 years. Potential risk factors associated with the winter season should be further studied in the future. After an orbital fracture repair, patients usually do not experience much pain and often return to their daily lives within a few days. It is important to educate patients that daily activities could lead to an increase in intraorbital pressure and ensure proper self-monitoring. Our case has contributed to the literature in this area by identifying daily activities that may increase the risk of retrobulbar hemorrhage. However, further studies on risk factors and prevention of retrobulbar hemorrhage are required.

\section{NOTES}

\section{Conflict of interest}

No potential conflict of interest relevant to this article was reported.

\section{Ethical approval}

The study was approved by the Institutional Review Board of Soonchunhyang University Cheonan Hospital (IRB No. 202012-039) and performed in accordance with the principles of the Declaration of Helsinki. Written informed consent was obtained.

\section{Patient consent}

The patient provided written informed consent for the publication and the use of his images.

\section{ORCID}

Da Woon Lee

Tae Hyung Kim

https://orcid.org/0000-0002-6969-5643

Hwan Jun Choi

https://orcid.org/0000-0001-9537-2330

Syeo Young Wee

https://orcid.org/0000-0002-0752-0389

https://orcid.org/0000-0002-1787-9715

\section{Author contribution}

Conceptualization: HJC. Formal analysis: THK. Funding acquisition: DWL. Project administration: SYW. Visualization: THK. Investigation: DWL. Resources: DWL, THK, SYW. Supervision: DWL. Validation: HJC. Writing - original draft: THK. Writing - review \& editing: THK.

\section{REFERENCES}

1. Cheon JS, Seo BN, Yang JY, Son KM. Retrobulbar hematoma in blow-out fracture after open reduction. Arch Plast Surg 
2013;40:445-9.

2. Christie B, Block L, Ma Y, Wick A, Afifi A. Retrobulbar hematoma: a systematic review of factors related to outcomes. J Plast Reconstr Aesthet Surg 2018;71:155-61.

3. Chen $\mathrm{CH}$, Chen CT, Huang F. Retrobulbar hematoma as a rare complication after secondary correction of enophthalmos. J Craniofac Surg 2009;20:963-7.

4. Beogo R, Coulibaly TA, Dolo MT, Traore I, Ouattara B, Diallo JW. Facial fracture-associated blindness. J Craniofac Surg 2018; 29:751-3.

5. Liu D. Blindness after blow-out fracture repair. Ophthalmic Plast Reconstr Surg 1994;10:206-10.

6. Park CW, Yang JY, Son KM, Cheon JS. The estimation of postoperative bleeding after reduction of blowout fracture: the effectiveness of negative-pressure drainage system. J Craniofac Surg 2015;26:873-5.

7. Eshraghi B, Torkashvand A, Katoozpour R, Anvari P. Delayed retrobulbar hemorrhage after reconstruction of inferior orbital wall fracture: a case report. J Egypt Ophthalmol Soc 2017;110: 141-2.

8. Michael I, Lily D, Matthew JT. Retrobulbar hematoma following orbital floor reconstruction. Eplasty 2013;13:ic45.

9. Omvik P. How smoking affects blood pressure. Blood Press 1996;5:71-7.

10. Wang S, Li M, Hua Z, Ye C, Jiang S, Wang Z, et al. Outdoor temperature and temperature maintenance associated with blood pressure in 438,811 Chinese adults. Blood Press 2017;26: 246-54.

11. Son KM, Park CW, Cheon JS. A case report of bilateral retrobulbar hemorrhage after lower blepharoplasty. Arch Craniofac Surg 2013;14:133-6.

12. Kang DH. Orbital wall restoring surgery with primary orbital wall fragments in blowout fracture. Arch Craniofac Surg 2019;20:347-53.

13. Matharu KS, Smith SV, Lee AG. Retrobulbar hemorrhage and prasugrel. Can J Ophthalmol 2016;51:e189-90. 